- Case Report

\title{
A Case of Primary Biliary Cirrhosis Mimicking Acute Hepatitis B in the Clinic, Republic of Korea
}

\author{
Woo Hyuk Kwon, Hong Min Park, Jeong Jun Park, Sung Hoon Lee, Yong Kyu Lee* \\ Department of Internal Medicine, Good Gang-An Hospital, Busan, Korea
}

Primary biliary cirrhosis (PBC) is a slowly progressive cholestatic autoimmune liver disease characterized by progressive bile duct injury. The most common symptoms of this disease include fatigue and pruritus. The diagnosis of PBC is based on cholestatic biochemical liver tests, presence of antimitochondrial antibodies, and characteristic histological biopsy findings. We report a case of a patient with PBS, who was initially suspected to be in the window period of hepatitis B by a private doctor in a local clinic based on the detection of isolated immunoglobulin $\mathrm{M}$ antibody against hepatitis B core antigen. The presence of this antibody is the most useful index in diagnosing acute hepatitis B (+) by immunoserological test. The final diagnosis of the patient in Good Gang-An Hospital was PBC through additional tests. The patient is receiving outpatient treatment.

Keywords: Cholestasis; Hepatitis; Immunoglobulins; Pruritus 


\section{INTRODUCTION}

Primary biliary cirrhosis (PBC) is an autoimmune hepatic disease characterized by chronic cholestasis that may gradually destruct the intrahepatic duct and cause portal venous inflammation and fibrosis. This condition frequently occurs in middle-aged women, with a prevalence of 2.7 to 40 per 100,000 persons. ${ }^{1)}$ PBC is characterized by the presence of antimitochondrial antibodies (AMAs) and high immunoglobulin (Ig) M level in the blood. ${ }^{2)}$ It may remain asymptomatic for an extended period of time or develop rapidly in a short period of time. IgM level can increase because of PBC. However, correlation between $\mathrm{PBC}$ and IgM antibody against hepatitis B core antigen (IgM anti-HBc antibody) has not been reported. Along with a literature review, we report a case of a $\mathrm{PBC}$ patient, who was initially suspected to be in the window period of hepatitis B in a local clinic because immunoserological test revealed isolated IgM anti-HBc antibodies (+).

\section{CASE REPORT}

A 35-year-old Asian man was referred to Good Gang-An Hospital mainly because of intermittent pruritus and fatigue that lasted for two months. The patient was suspected to be in the window period of hepatitis B based on the detection of IgM anti-HBc antibody (+) with increased aspartate transaminase (AST) and alanine transaminase (ALT) levels in his blood about a month ago. He had been drinking approximately a bottle of beer once a month but does not smoke. His family history was not significant. At the time of his visit to this hospital, his blood pressure was 120/80 mm Hg; pulse rate, 76/min; respiration rate, $20 / \mathrm{min}$; and temperature, $36.5^{\circ} \mathrm{C}$. Moreover, the patient was con-

Table 1. Patient's initial laboratory data

\begin{tabular}{lcc}
\hline \multicolumn{1}{c}{ Variable } & Value & Reference range \\
\hline Hemoglobin (g/dL) & 14.6 & $11-16$ \\
White blood cell (cells/mcL) & 4,880 & $3,600-9,500$ \\
Platelet (cells/mcL) & 181,000 & $150,000-450,000$ \\
Total protein (g/dL) & 9.2 & $6.4-8.3$ \\
Albumin (g/dL) & 4.4 & $3.8-5.3$ \\
Total bilirubin (mg/dL) & 0.4 & $0.2-1.2$ \\
Direct bilirubin (mg/dL) & 0.1 & $0-0.4$ \\
Blood urea nitrogen (mg/dL) & 13.9 & $7-20$ \\
Creatinine (mg/dL) & 1.0 & $0.7-1.3$ \\
Lactate dehydrogenase (IU/L) & 518 & $263-450$ \\
Aspartate transaminase (IU/L) & 107 & $8-38$ \\
Alanine transaminase (IU/L) & 104 & $5-43$ \\
Alkaline phosphatase (IU/L) & 1,431 & $35-105$ \\
Gamma-glutamyl transpeptidase (IU/L) & 813 & $15-75$ \\
IgM anti-hepatitis B core antibody & + & - \\
Hepatitis B surface Ag/Ab & $-/-$ & $-/-$ \\
Hepatitis B e Ag/Ab & $-/-$ & $-/-$ \\
Hepatitis C virus Ab & - & - \\
Anti-hepatitis A virus IgM/lgG & $-/-$ & - \\
Hepatitis B virus DNA quantification & - & - \\
\hline
\end{tabular}

Ig, immunoglobulin; Ag, antigen; Ab, antibody. scious and had no specific symptom but fatigue. His breath sound was normal during chest auscultation; neither whole body jaundice nor icteric sclera was observed. No tactile mass was noted in the abdominal region; neither abdominal pain nor abdominal fluid was found. The peripheral blood test showed that his hemoglobin was $14.6 \mathrm{~g} / \mathrm{dL}$ (reference range, 11-16 g/dL); white blood cell count, 4,880 cells $/ \mathrm{mcL}$ (reference range, 3,600-9,500 cells/mcL); and platelet, 181,000 cells/ mcL (reference range, 150,000-450,000 cells/mcL). The biochemical test revealed the following: total protein, $9.2 \mathrm{~g} / \mathrm{dL}$ (reference range, 6.4-8.3 g/dL); albumin, $4.4 \mathrm{~g} / \mathrm{dL}$ (reference range, 3.8-5.3 g/dL); AST, $107 \mathrm{IU} / \mathrm{L}$ (reference range, 8-38 IU/L); ALT, $104 \mathrm{IU} / \mathrm{L}$ (reference range, 5-43 IU/L); alkaline phosphatase (ALP), 1,431 IU/L (reference range, 35-105 IU/L); gamma-glutamyl transpeptidase (GGT), 813 IU/L (reference range, 15-75 IU/L); total bilirubin, $0.4 \mathrm{mg} / \mathrm{dL}$ (reference range, $0.2-1.2 \mathrm{mg} / \mathrm{dL}$ ); direct bilirubin, $0.1 \mathrm{mg} / \mathrm{dL}$ (reference range, $0-0.4 \mathrm{mg} /$ $\mathrm{dL}$ ); blood urea nitrogen, $13.9 \mathrm{mg} / \mathrm{dL}$ (reference range, $7-20 \mathrm{mg} / \mathrm{dL}$ ); creatinine, $1.0 \mathrm{mg} / \mathrm{dL}$ (reference range, $0.7-1.3 \mathrm{mg} / \mathrm{dL}$ ); and lactate dehydrogenase, $518 \mathrm{IU} / \mathrm{L}$ (reference range, 263-450 IU/L). The immunoserological test found hepatitis B surface (HBs) antigen/antibody (-/-), hepatitis B e (HBe) antigen/antibody (-/-), hepatitis C virus antibody (-), anti-hepatitis A virus (HAV) IgM (-), anti-HAV IgG (-), IgM anti-HBc antibody (+), and hepatitis B virus (HBV) DNA quantification (-) (Table 1). He was diagnosed with PBC because of his increased ALP and GGT levels in the blood test and increased IgM/IgG $(518 / 1,740 \mathrm{mg} / \mathrm{dL})$ in the additional blood test; he was also both AMA and antinuclear antibody (ANA) positive. Nothing significant was noted on his chest and simple abdominal X-ray. Moreover, no abnormal findings were observed; however, an approximately $2 \mathrm{~cm}$ hepatic cyst was found through abdominal computed tomography (Figure 1). The development of the disease and screen associated autoimmune hepatitis was assessed. Liver biopsy found intrahepatic bile duct injury and

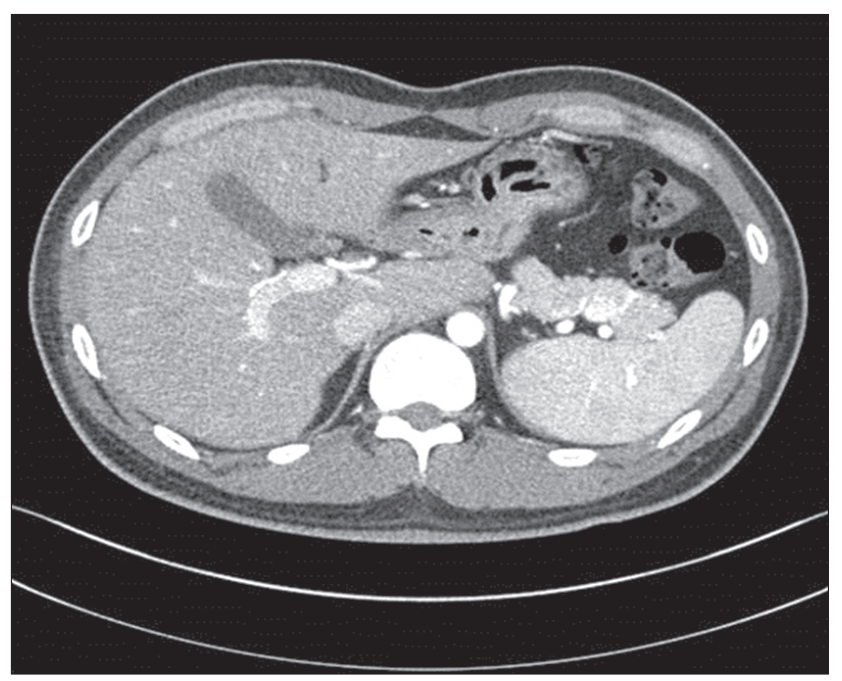

Figure 1. Portal phase-enhanced computed tomography performed on the second hospital day showed no significant abnormal finding in the biliary tract, except for the single hepatic cyst on the left lateral segment. 

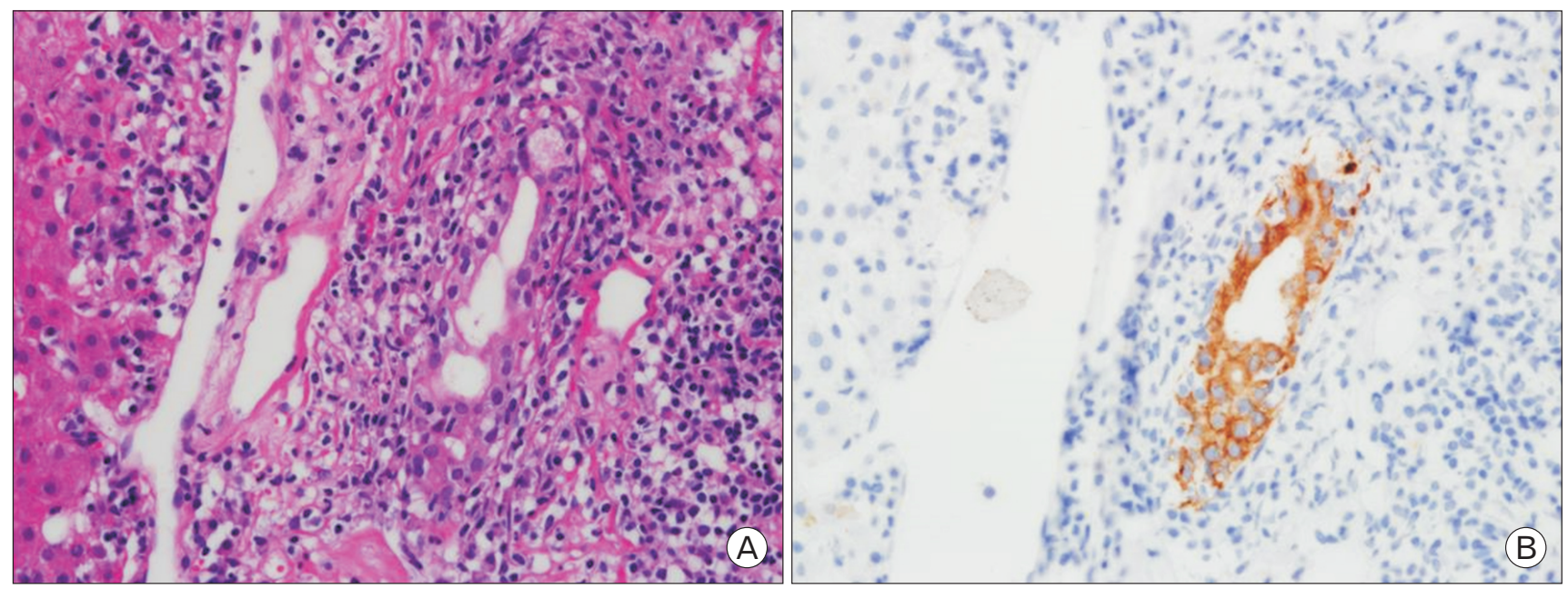

Figure 2. (A) Portal area was expanded by lymphoplasma cell infiltration and showed mild ductal damage. The epithelial cells of the interlobular duct were vacuolated and disorganized (H\&E, $\times 400)$. (B) In the immunohistochemical stain of CK7 (biliary epithelial marker), the intraepithelial infiltration of lymphocytes was recognizable (immunoperoxidase, original magnification, $\times 400$ ).

Table 2. Biochemical changes in a primary biliary cirrhosis patient for 6 months

\begin{tabular}{lcccc}
\hline & At admission & 1 month later & 3 months later & 6 months later \\
\hline Aspartate transaminase (IU/L) & 107 & 33 & 34 & 26 \\
Alanine transaminase (IU/L) & 104 & 18 & 18 & 16 \\
Alkaline phosphatase (IU/L) & 1,431 & 452 & 350 & 288 \\
Gamma-glutamyl transpeptidase (IU/L) & 813 & 173 & 107 & 89 \\
Immunoglobulin M anti-hepatitis B core antibody & + & & - \\
\hline
\end{tabular}

epithelial cell infiltration in the lymphocytes, both of which characterize $\mathrm{PBC}$, but did not indicate severe fibrosis or any condition that suggested autoimmune hepatitis (Figure 2A, B). Based on AMA and ANA $(+)$ and the blood test results, which suggested a cholestatic disease, he was diagnosed with PBC. The patient was administered with a high dose of ursodesoxycholic acid (UDCA, $900 \mathrm{mg} / \mathrm{d}$ ) for three months. After the treatment, both fatigue and pruritus decreased, and the AST, ALT, ALP, and GGT were 34, 18, 350, and 107 IU/L, respectively. Six months later, the patient exhibited lower ALP and GGT levels and negative IgM anti-HBc antibody in the follow-up test (Table 2).

\section{DISCUSSION}

The most useful index in diagnosing acute hepatitis B is IgM anti-HBc antibody, which increased for the first two to four weeks after the HBV infection and then began to decrease gradually. This antibody was detected in the blood for about six months after the acute HBV infection and was the only serological index that could determine the recent HBV infection during the window period between the disappearance of HBs antigen and the appearance of HBs antibody. If IgM anti-HBc antibody is separately detected, the patient can be suspected to have anti-HBc false-positive, to be in the window period, to be in the convalescent stage after HBV infection, or to have latent HBV injection. This case was considered anti-HBc antibody false-positive because HBs antigen/antibody, $\mathrm{HBe}$ antigen, and anti-HBe negative were maintained and HBV DNA was not detected during the six months of follow-up. Ninety percent of the PBC patients are female, and this condition frequently occurs in those in their fifties and sixties. Pruritus and fatigue are two of the most common initial symptoms, and $\mathrm{PBC}$ has recently been detected more frequently in its early stages during medical check-up, with $50 \%$ to $60 \%$ of all the cases detected by chance in liver function test. ${ }^{3)} \mathrm{PBC}$ is a cholestatic autoimmune disease, with $90 \%$ to $95 \%$ of all the cases being AMA $(+)^{4,5)}$ and approximately $50 \%$ being ANA (+). It is accompanied by the increase in ALP and GGT levels, which characterize cholestatic liver diseases, and the increase in IgM level in the blood in most cases. This phenomenon is attributed to the lymphocytes that are deposited around damaged bile canaliculi, which usually generate IgM in PBC patients. ${ }^{6)}$ UDCA is the only medicine for PBC and has been officially approved by the Food and Drug Administration. This medicine may be administered at a dose of 13-15 $\mathrm{mg} / \mathrm{kg} / \mathrm{d}$. Its therapeutic effects may be determined through the results from biochemical tests (ALP, bilirubin, etc.) in six to nine months in most cases and those cases with therapeutic effects may have good long-term prognoses. UDCA may reduce the need of liver transplantation, improve the survival rate, and control the development of varicose veins. ${ }^{7,8)}$ Liver transplantation may be considered when $\mathrm{PBC}$ is accompanied by pruritus, which cannot be controlled by medication, has severe osteoporosis, suffers hepatic failure because of the condi- 
tion, or feels badly fatigued. ${ }^{9)}$ The patient in this case was suspected to be in the window period of acute hepatitis B because he had non-specific symptoms, such as continuous fatigue and intermittent pruritus, and compromised liver functions. Moreover, the patient was IgM antiHBc antibody (+). However, he underwent AMA and ANA examination and liver biopsy for cholestatic hepatitis because his blood test showed an increase in the ALP and GGT levels. He was diagnosed with PBC based on the AMA and ANA (+) finding and the liver biopsy results. The anti-HBc false-positive finding is probably due to the increased IgM level in blood, which leads to antibodies with cross reactivity in $\mathrm{HBc}$ antigen and their non-specific binding. The patient in this case was IgM anti-HBc antibody (+) in the immunoserological test when he visited the hospital. His ALP and GGT levels then improved with UDCA administration. After six months, his immunoserological test result showed IgM anti-HBc antibody (-). This result is probably due to the decreased IgM level in the blood with the favorable turn of the condition after the treatment. Isolated IgM anti-HBc antibody (+) is generally found in immunoserological test. The condition may be treated in relation to viral hepatitis: the HBV window period, the convalescent stage after HBV infection, or latent HBV infection. In this case, the patient was also initially strongly suspected to be in the window period of acute hepatitis B because he was a young man with IgM anti-HBc antibody (+) in two consecutive tests. Then, he was suspected of cholestatic hepatitis because HBV DNA was undetected and the ALP and GGT levels increased remarkably and was diagnosed with $\mathrm{PBC}$ in the additional test. The increased IgM level in the blood can likely generate antibodies with cross reactivity in $\mathrm{HBc}$ antigen, which may be false-positive. However, correlation between PBC and anti HBc-IgM antibody or the frequency of being false-positive has not been reported. Thus, further research should be conducted on these factors.

In conclusion, IgM anti-HBc antibody is the most useful index in diagnosing acute hepatitis $\mathrm{B}$. This antibody increases for the first two to four weeks after the HBV infection. However, it is necessary to consider the possibility of PBC when an acute hepatitis patient has liver dysfunction, isolated IgM anti-HBc antibody (+), and increased ALP and GGT levels. If a patient with abnormal findings from immunoserological test sees the ALP and GGT levels raised, it is helpful in seeing a favorable turn of the condition and better prognoses to perform additional blood test for cholestatic hepatitis along with liver biopsy as needed to start proper treatment on the basis of correct diagnosis.

\section{CONFLICT OF INTEREST}

No potential conflict of interest relevant to this article was reported.

\section{REFERENCES}

1. Kim WR, Lindor KD, Locke GR 3rd, Therneau TM, Homburger HA, Batts KP, et al. Epidemiology and natural history of primary biliary cirrhosis in a US community. Gastroenterology 2000;119:1631-6.

2. Gershwin ME, Mackay IR, Sturgess A, Coppel RL. Identification and specificity of a cDNA encoding the $70 \mathrm{kd}$ mitochondrial antigen recognized in primary biliary cirrhosis. J Immunol 1987;138:3525-31.

3. Lee YM, Kaplan MM. The natural history of PBC: has it changed? Seminars in liver disease. New York (NY): Thieme Medical Publishers Inc.; 2005.

4. Walker JG, Doniach D, Roitt IM, Sherlock S. Serological tests in diagnosis of primary biliary cirrhosis. Lancet 1965;1:827-31.

5. Berg PA, Klein R, Lindenborn-Fotinos J. Antimitochondrial antibodies in primary biliary cirrhosis. J Hepatol 1986;2:123-31.

6. Walker G, Doniach D. Antibodies and immunoglobulins in liver disease. Gut 1968;9:266-9.

7. Kaplan MM, Gershwin ME. Primary biliary cirrhosis. N Engl J Med 2005;353:1261-73.

8. Lindor KD, Gershwin ME, Poupon R, Kaplan M, Bergasa NV, Heathcote EJ, et al. Primary biliary cirrhosis. Hepatology 2009;50:291-308.

9. Neuberger J. Liver transplantation for primary biliary cirrhosis. Autoimmun Rev 2003;2:1-7. 